\title{
A Skew Extension of the Multivariate Generalized T Distributions with Applications
}

\author{
A.M.Fares $^{1 *}$, V.V.Haragopal ${ }^{2}$ \\ ${ }^{1}$ Higher Institute For Administrative Development, Damascus University, Halboni, Damascus, Syria \\ ${ }^{1}$ Department of Statistics, Osmania University, Hyderabad, India \\ ${ }^{2}$ Department of Mathematics, Birla Institute of Technology and Science, Pilani-Hyderabad Campus, Hyderabad, India \\ ${ }^{2}$ Formally, Department of Statistics, Osmania University, Hyderabad, India \\ "Corresponding Author: fares26285@hotmail.com, Tel.: +91-91774-58563
}

Available online at: www.isroset.org

Received: 06/Jun/2018, Revised: 13/Jun/2018, Accepted: 21/Jun/2018, Online: 30/Jun/2018

\begin{abstract}
We propose an asymmetric extension of Arslan multivariate generalization of the Generalized $\mathrm{T}$ distributions introduced by McDonald and Newey, the proposed Skew Multivariate Generalized T distribution (MSGT) includes the skew multivariate Normal distribution of Azzalini and Valle as well as the skew multivariate $t$ distribution introduced by Azzalini and Capitanio, besides, MSGT contains many other distributions as a limiting or special cases, a stochastic representation of MSGT random vector has been obtained along with a simulation study, the flexibility added of this distribution makes it a potential candidate in modeling multidimensional data of different tail behaviors for providing an outstanding fit which we have practically illustrated by using this distribution for modeling multivariate data of the American financial market.
\end{abstract}

Keywords-Multivariate; Skew; Generalized T-distribution.

\section{INTRODUCTION}

Recently, there has been a considerable interest in developing more flexible statistical distributions at a multidimensional level, modifying a symmetric univariate density around origin to introduce skewness emerged frequently in the literature and was a key development for adding more flexibility to the fit, Azzalini [1] has introduced the Skew normal distribution by combining the standard normal density of a random variable with its distribution function after rescaling it with a real quantity that is the skewness parameter, Azzalini and Valle [2] have extended the idea to a multivariate level and introduced the skew multivariate Normal distribution which led later to several general procedures for forming skew multivariate distributions among them perturbation of symmetry by Azzalini and Capitanio [3] in which the multivariate-t distribution was obtained, Genton [4] defined generalized skew-elliptical distributions that include the later ones, the formula of the skew generalized $\mathrm{T}$ distribution proposed in this paper is basically motivated by the work of both last two authors [3] and [4], where we define a skew elliptical density as an extension of the multivariate generalized $\mathrm{T}$ distribution of Arslan [5] which covers the multivariate generalization of the generalized-t distribution defined by McDonald and Newey [6].

The Remainder of this paper is organized as follows, in the next section we reparametrize the univariate Generalized $\mathrm{T}$ distribution (GT) introduced by [6], followed by the same for its distribution function introduced by Nadarajah [7] and its multivariate generalization introduced by Arslan [5], in section III we define the Skew Multivariate Generalized T distribution (MSGT) then we give a Stochastic representation in section IV, we apply this distribution on the American market returns in section $\mathrm{V}$ then we discuss the results in section VI and conclude in section VII.

\section{SOME ADJUSTMENTS}

We start the construction of the multivariate skew generalized $\mathrm{T}$ distribution (MSGT) by considering the generalized $\mathrm{T}$ distribution of [6] but with another parameterization given by: 


$$
f_{G T}(x ; m, s, \alpha, v)=\frac{\alpha}{2 s v^{\frac{1}{a}} \beta\left(\frac{v}{\alpha}, \frac{1}{\alpha}\right)\left(1+\frac{|x-m|^{\alpha}}{v s^{\alpha}}\right)^{\frac{v+1}{\alpha}}}
$$

$x, m \in R \quad s, v, \alpha>0$

It can be shown that the above density with $m=0$ and $s=1$ is a mixture of the exponential power distribution (PE) of Box [8] with a scale parameter distributed as inverse generalized gamma (IGG) of Hoq [9] with inverse scale $\theta=v^{\frac{1}{\alpha}}$, so we have

$$
f_{P E}(\mathrm{x} ; \sigma, \alpha)=\frac{\alpha e^{-\left(\frac{|\mathrm{x}|}{\sigma}\right) \alpha}}{2 \sigma \Gamma \frac{1}{\alpha}}
$$

And

$$
f_{I G G}\left(\sigma ; q=v, \theta=v^{\frac{1}{\alpha}}, \beta=\alpha\right)=\frac{\alpha v^{\frac{v}{\alpha}} e^{-\frac{v}{\sigma^{\alpha}}}}{\Gamma \frac{v}{\alpha} \sigma^{v+1}}
$$

$f_{G T}(\mathrm{x} ; 0,1, \alpha, v)=\int_{0}^{\infty} f_{P E}(\mathrm{x} ; \sigma, \alpha) f_{I G G}\left(\sigma ; q=v, \theta=v^{\frac{1}{\alpha}}, \beta=\alpha\right) d \sigma$

The cumulative distribution function of (GT) density was derived by [7], hence the CDF of the (GT) density given in (1.1) can accordingly be given by:

$$
F_{G T}(\mathrm{x}, m, s, a, v)=\frac{1}{2}\left[1+\operatorname{sign}(\mathrm{x}-m) \mathrm{I}_{U}\left(\frac{v}{\alpha}, \frac{1}{\alpha}\right)\right]
$$

$$
U=1-\frac{1}{\left(\frac{|x-m|}{s v^{\frac{1}{a}}}\right)^{a}+1}
$$

And

$\mathrm{I}_{u}(a, b)=\frac{\int_{-\infty}^{u} w^{a-1}(1-w)^{b-1}}{\beta(a, b)} d w$
Which is the regularized Beta function.

Now, we define the multivariate generalized $\mathrm{T}$ distribution (MGT) of [5] in such a parameterization that is analogous to its univariate density function given in (1.1) as follows:

Let $\mathrm{X}$ be a d-dimensional random variable distributed as MGT then its PDF is given by:

$$
f_{M G T}(\mathrm{X}, \zeta, \Omega, \alpha, v)=\frac{\alpha \Gamma\left(\frac{d}{2}\right)}{2 \pi^{\frac{d}{2}} v^{\frac{d}{\alpha}} \beta\left(\frac{v}{\alpha}, \frac{d}{\alpha}\right)|\Omega|^{\frac{1}{2}}\left(1+\frac{Q^{\frac{\alpha}{2}}}{v}\right)^{\frac{v+d}{\alpha}}}
$$

Where $\zeta \in R^{d}, \Omega$ is a positive-definite symmetric matrix and $Q=(\mathrm{X}-\zeta)^{T} \Omega^{-1}(\mathrm{X}-\zeta)$.
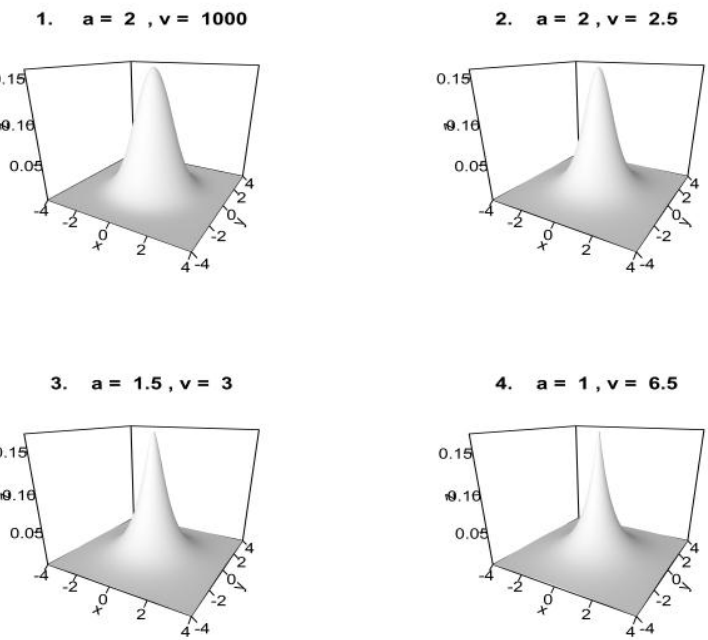

Figure 1. Bivariate Generalized $\mathrm{T}$ densities with identical locations and unit scale matrices but different shape parameters, where the first panel represents an approximation of the bivariate Normal distribution (since $\alpha=2$ and $v$ is large enough) and the second one is the bivariate $t$ distribution whereas the fourth one shows the bivariate GDL density, to illustrate how the flatness of the peak decreases as $\alpha$ decreases, also how the tails become heavier with smaller $v$.

- For $\alpha=2$ the above density in (1.8) reduces to the multivariate $\mathrm{t}$ distribution (MT) which approaches to the multivariate Normal distribution (MN) for $v \rightarrow \infty$.

- For $v \rightarrow \infty$ The above density in (1.8) approaches to the multivariate power exponential distribution 
(MPE) defined by Gomez et al [10] but with slightly different parameterization given by:

$$
f_{P E}(\mathrm{X} ; \zeta, \Sigma, \alpha)=\frac{\alpha^{1-\frac{d}{2}} \Gamma\left(\frac{d}{2}\right) e^{-\left(\frac{Q}{\alpha}\right)^{\frac{\alpha}{2}}}}{2 \pi^{\frac{d}{2}} \Gamma\left(\frac{d}{\alpha}\right)|\Omega|^{\frac{1}{2}}}
$$

Which includes the multivariate Normal distribution for $\alpha=2$ and the multivariate Laplace distribution for $\alpha=1$.

- Consider the univariate generalized double Lomax distribution (GDL) defined by Fares and Haragopal [11] with scale parameter $=(v s)$,

$$
f_{G D L}(\mathrm{x} ; m, s, v)=\frac{1}{2 s\left(1+\frac{|\mathrm{x}-m|}{v s}\right)^{v+1}}
$$

Then the (MGT) density in (1.8) reduces (for $\alpha=1$ ) to the multivariate generalized double Lomax distribution (MGDL) which is the multivariate generalization of (GDL) given in (1.10), also MGDL approaches to the multivariate Laplace distribution for $v \rightarrow \infty$.

As the MGT distribution belongs to the family of elliptically contoured distributions, according to Fang and Zhang [12], the random vector X of PDF defined in (1.8) has the same distribution as:

$$
\begin{aligned}
\zeta+R A^{T} U^{(d)} & \\
h_{R}(r) & =G B 2\left(r ; a=\alpha, b=v^{\frac{1}{\alpha}}, p=\frac{d}{\alpha}, q=\frac{v}{\alpha}\right) \\
& =\frac{\alpha r^{d-1}}{v^{\frac{d}{\alpha}} \beta\left(\frac{v}{\alpha}, \frac{d}{\alpha}\right)\left(1+\frac{r^{\alpha}}{v}\right)^{\frac{v+d}{\alpha}}}
\end{aligned}
$$

Where $U^{(d)}$ is a random vector uniformly distributed on the unit sphere in $R^{d}, A$ is a square matrix with $d$ order such that $A^{T} A=\Omega$ and $\mathrm{R}$ is an absolutely continuous non negative random variable, independent from $U^{(d)}$, whose density function is:

$$
\begin{aligned}
h_{R}(r) & =G B 2\left(r ; a=\alpha, b=v^{\frac{1}{\alpha}}, p=\frac{d}{\alpha}, q=\frac{v}{\alpha}\right) \\
& =\frac{\alpha r^{d-1}}{v^{\frac{d}{\alpha}} \beta\left(\frac{v}{\alpha}, \frac{d}{\alpha}\right)\left(1+\frac{r^{\alpha}}{v}\right)^{\frac{v+d}{\alpha}}}
\end{aligned}
$$

Where GB2(.) is the PDF of the Generalized beta distribution of second kind defined by McDonald and Xu [13], with $k^{\text {th }}$ order moment given by:

$$
\mathrm{E}\left(R^{k}\right)=\frac{v^{\frac{k}{\alpha}} \beta\left(\frac{d+k}{\alpha}, \frac{v-k}{\alpha}\right)}{\beta\left(\frac{v}{\alpha}, \frac{d}{\alpha}\right)}, \quad v>k
$$

\section{SKEW EXTENSION OF THE MULTIVARIATE GENERALIZED T DISTRIBUTION:}

We propose a skew extension of MGDL by using Genton [4] skewing procedure by combining the symmetric elliptical multivariate density of MGDL with a skewing function defined below and the resulting distribution is a class of generalized skew-elliptical distributions given by:

$$
G S E(X)=2 f(X) \pi(X)
$$

Where $f($.$) is an elliptical density and \pi($.$) is a skewing$ function that satisfies $0 \leq \pi(X) \leq 1$ and $\pi(-X)=1-\pi(X)$, we define the multivariate skew generalized T distribution (MSGT) as a special case of (1.15) replacing $f($.$) by f_{M G T}($.$) and \pi($.$) by F_{G T}($.$) as$ follows:

$$
\begin{aligned}
& f_{M S G T}(X, \zeta, \Omega, \lambda, \alpha, v)=2 \times f_{M G T}(X, \zeta, \Omega, \alpha, v) \\
& \times F_{G T}\left(\lambda^{T} \omega^{-1}(X-\zeta)\left(\frac{v+d}{v+Q}\right)^{\frac{1}{\alpha}}, 0,1, \alpha, v+d\right)
\end{aligned}
$$

Where $\lambda \in R^{d} \quad$ is the skewness parameter, $\omega=\{\operatorname{diag}(\Omega)\}^{\frac{1}{2}}, f_{M G T}(\mathrm{X}, \zeta, \Omega, \alpha, v)$ is defined in 
(1.8), and the skewing function here $\pi(X)=F_{G T}\left(\lambda^{T} \omega^{-1}(X-\zeta)\left(\frac{v+d}{v+Q}\right)^{\frac{1}{\alpha}}, 0,1, \alpha, v+d\right)$

is the univariate GT distribution function defined in (1.5).
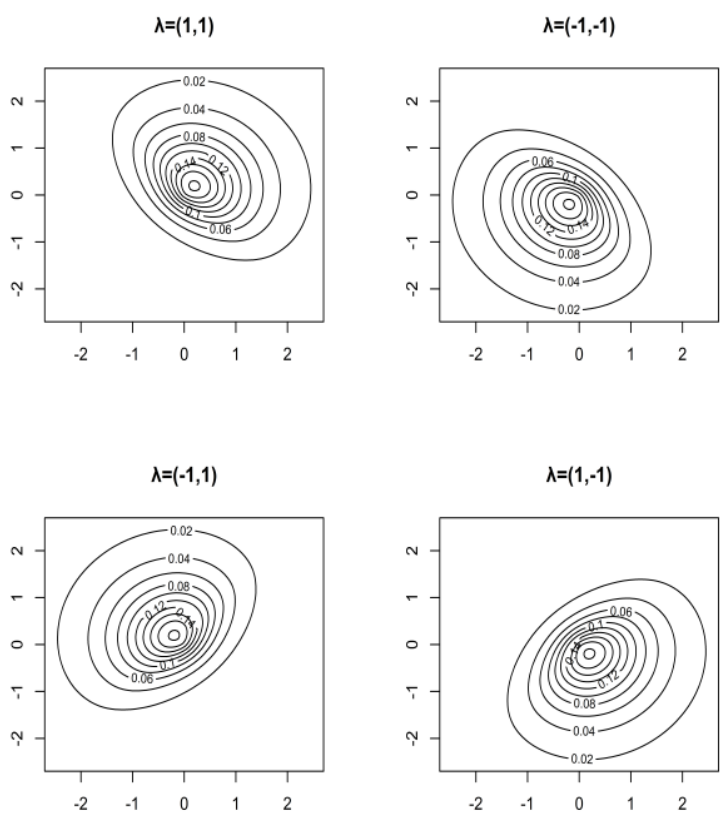

Figure 2. Contur plot of Bivariate SGT Densities with identical locations, unit scale matrices and shape parameters $\alpha=1.5$ and $v=3$, but different skewness parameters, where we can see how the sings of the skewness vector components shift the density's tails to the corresponding quarter.

- For $\alpha=2$ the above density in (1.16) reduces the multivariate skew $\mathrm{t}$ distribution (MST) defined by [4] which approaches to multivariate skew Normal distribution (MSN) of [5] for $v \rightarrow \infty$.

- For $v \rightarrow \infty$ the above density in (1.16) approaches to the multivariate skew power exponential distribution (MSPE) Which includes the multivariate skew Normal distribution for $\alpha=2$ and the multivariate skew Laplace distribution (MSLaplace) for $\alpha=1$.

- MSGT reduces (for $\alpha=1$ ) to the multivariate skew generalized double Lomax distribution (MSGDL) which also approaches to MSLaplace for $v \rightarrow \infty$.

\section{STOChaSTIC REPRESENTATION AND SIMULATION}

We obtain a stochastic representation of MSGT random vector following the skew-symmetric representation of Wang and Boyer [14] as follows:
Let $\mathrm{Y}$ be a continuous random vector with $\operatorname{PDF} f(y)$ that is symmetric around $0_{d}$. Let $\mathrm{U}$ be a uniform random variable on $(0,1)$, independent of $Y$.

The random vector $\mathrm{X}$ with PDF (1.16) can be simulated by the following representation:

$X=\left\{\begin{array}{c}Y+\zeta \text { if } \quad U<\pi(Y) \\ -Y+\zeta \text { if } \quad U>\pi(Y)\end{array}\right.$

Replacing $\pi(y)$ by $F_{G T}($.$) in the above equation we get$ $X \sim \operatorname{MSGT}(\zeta, \Omega, \lambda, \alpha, v)$ represented by:

$X=\zeta+Y \times \operatorname{sign}\left(F_{G T}\left(\lambda^{T} \omega^{-1}(X-\zeta)\left(\frac{v+d}{v+Q}\right), 0,1, \alpha, v+d\right)-U\right)$

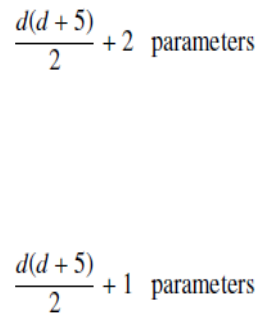

$\frac{d(d+5)}{2} \quad$ parameter

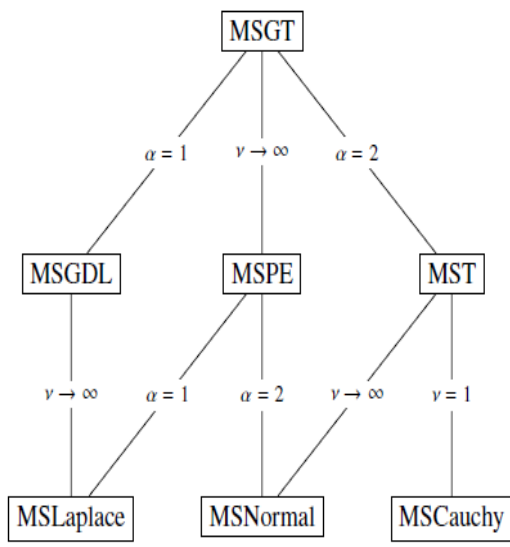

Figure 3. Some multivariate skew distributions as either special or limiting cases of MSGT distribution introduced in this paper

The Monte Carlo experiment has been performed with 500 iterations and repeated for different sample sizes to evaluate the Maximum likelihood Estimations (MLE) in terms of the estimates means and standard deviations, we consider the values of the true parameters to be:

$$
(\zeta, \Omega, \lambda, \nu, \alpha)=\left(\left[\begin{array}{l}
1 \\
2
\end{array}\right],\left[\begin{array}{ll}
4 & 1 \\
1 & 2
\end{array}\right],\left[\begin{array}{r}
-1 \\
1
\end{array}\right], 4.5,1.5\right)
$$

Where the random vectors generating process is of the stochastic representations defined in (1.19). The simulation results are displayed in Table 1, the consistency of the MLE is well pronounced as the bias and standard deviations decrease with larger sample sizes. 
Table 1. MSGT Simulation results.

\begin{tabular}{|c|c|c|c|c|c|c|c|c|c|c|}
\hline $\mathbf{n}$ & $\xi_{1}$ & $\xi_{2}$ & $\hat{v}$ & $\hat{\alpha}$ & $\dot{\lambda}_{1}$ & $\dot{\lambda}_{2}$ & \multicolumn{2}{|c|}{$\hat{\Omega}$} & \multicolumn{2}{|c|}{$S D_{\Omega}$} \\
\hline 100 & $\begin{array}{r}0.9061 \\
(0.7131)\end{array}$ & $\begin{array}{c}2.0706 \\
(0.4984)\end{array}$ & $\begin{array}{r}17.7227 \\
(18.5591)\end{array}$ & $\begin{array}{r}1.4678 \\
(0.6838)\end{array}$ & $\begin{array}{r}-1.0131 \\
(0.7429)\end{array}$ & $\begin{array}{c}0.9676 \\
(0.7293)\end{array}$ & $\begin{array}{l}3.9485 \\
1.0922\end{array}$ & $\begin{array}{l}1.0922 \\
1.9636\end{array}$ & $\begin{array}{l}(2.1060) \\
(0.7705)\end{array}$ & $\begin{array}{l}(0.7705) \\
(1.0054)\end{array}$ \\
\hline 500 & $\begin{array}{r}0.9825 \\
(0.2853)\end{array}$ & $\begin{array}{c}2.0130 \\
(0.2168)\end{array}$ & $\begin{array}{r}7.0629 \\
(8.1811)\end{array}$ & $\begin{array}{c}1.4638 \\
(0.2897)\end{array}$ & $\begin{array}{r}-1.0060 \\
(0.2344)\end{array}$ & $\begin{array}{r}0.9633 \\
(0.2511)\end{array}$ & $\begin{array}{l}3.9191 \\
0.9955\end{array}$ & $\begin{array}{l}0.9955 \\
1.9613\end{array}$ & $\begin{array}{l}(0.7995) \\
(0.2400)\end{array}$ & $\begin{array}{l}(0.2400) \\
(0.3931)\end{array}$ \\
\hline 1000 & $\begin{array}{r}1.0156 \\
(0.1918)\end{array}$ & $\begin{array}{c}2.0158 \\
(0.1453)\end{array}$ & $\begin{array}{r}5.1875 \\
(3.1337)\end{array}$ & $\begin{array}{c}1.4791 \\
(0.1763)\end{array}$ & $\begin{array}{r}-1.0366 \\
(0.1646)\end{array}$ & $\begin{array}{r}0.9761 \\
(0.1685)\end{array}$ & $\begin{array}{l}3.9259 \\
0.9893\end{array}$ & $\begin{array}{l}0.9893 \\
1.9633\end{array}$ & $\begin{array}{l}(0.4755) \\
(0.1546)\end{array}$ & $\begin{array}{l}(0.1546) \\
(0.2324)\end{array}$ \\
\hline 2500 & $\begin{array}{r}1.0000 \\
(0.1231)\end{array}$ & $\begin{array}{c}2.0028 \\
(0.0845)\end{array}$ & $\begin{array}{r}4.6111 \\
(0.6808)\end{array}$ & $\begin{array}{l}1.5026 \\
(0.1110)\end{array}$ & $\begin{array}{r}-1.0219 \\
(0.1069)\end{array}$ & $\begin{array}{r}0.9779 \\
(0.1007)\end{array}$ & $\begin{array}{l}3.9833 \\
1.0023\end{array}$ & $\begin{array}{l}1.0023 \\
1.9891\end{array}$ & $\begin{array}{l}(0.2896) \\
(0.1001)\end{array}$ & $\begin{array}{l}(0.1001) \\
(0.1395)\end{array}$ \\
\hline 5000 & $\begin{array}{r}0.9991 \\
(0.0932)\end{array}$ & $\begin{array}{c}2.0008 \\
(0.0682)\end{array}$ & $\begin{array}{r}4.5793 \\
(0.4592)\end{array}$ & $\begin{array}{c}1.4992 \\
(0.0758)\end{array}$ & $\begin{array}{r}-1.0229 \\
(0.0744)\end{array}$ & $\begin{array}{r}0.9760 \\
(0.0747)\end{array}$ & $\begin{array}{l}3.9943 \\
1.0028\end{array}$ & $\begin{array}{l}1.0028 \\
1.9938\end{array}$ & $\begin{array}{l}(0.2092) \\
(0.0698)\end{array}$ & $\begin{array}{l}(0.0698) \\
(0.0961)\end{array}$ \\
\hline 10000 & $\begin{array}{r}1.0065 \\
(0.0634)\end{array}$ & $\begin{array}{l}2.0041 \\
(0.0458)\end{array}$ & $\begin{array}{r}4.5271 \\
(0.3475)\end{array}$ & $\begin{array}{c}1.5000 \\
(0.0575)\end{array}$ & $\begin{array}{l}-1.0261 \\
(0.0522)\end{array}$ & $\begin{array}{r}0.9773 \\
(0.0514)\end{array}$ & $\begin{array}{l}3.9994 \\
1.0007\end{array}$ & $\begin{array}{l}1.0007 \\
1.9954\end{array}$ & $\begin{array}{l}(0.1418) \\
(0.0490)\end{array}$ & $\begin{array}{l}(0.0490) \\
(0.0687)\end{array}$ \\
\hline
\end{tabular}

\section{Applications}

MSGT and MST along with their symmetric versions have been fitted to a data set consisting of 5032 pair of daily logreturn belonging to the American indices, also we have used the multivariate Generalized Hyperbolic distribution (MGHYP) for modeling the same data.

The Maximum Likelihood Estimation has been applied using $\mathrm{R}$ programing Language to obtain the parameters' estimates, Table 2 shows the results of this data modeling. In brief,

Table 2. Parameter estimates for NASDAQ and S\&P500 daily returns.

\begin{tabular}{|c|c|c|c|c|c|c|c|c|c|c|}
\hline \multirow{2}{*}{ Model } & \multicolumn{8}{|c|}{ MLE } & \multicolumn{2}{|c|}{ Information } \\
\hline & $\zeta_{1}$ & $\zeta_{2}$ & $\alpha$ & $v$ & $\lambda_{1}$ & $\lambda_{2}$ & \multicolumn{2}{|c|}{$\Omega$} & Log-likelihood & BIC \\
\hline \multirow[b]{2}{*}{ MGT } & & & & & & & 0.0000593 & 0.0000417 & \multirow[b]{2}{*}{-34346} & \multirow[b]{2}{*}{.68632 } \\
\hline & 0.00107 & 0.00068 & 1.19 & 4.27 & . & . & 0.0000417 & 0.0000361 & & \\
\hline \multirow[b]{2}{*}{ MSGT } & & & & & & & 0.0000618 & 0.0000428 & \multirow[b]{2}{*}{-34359} & \multirow[b]{2}{*}{-68642} \\
\hline & 0.00291 & 0.00133 & 1.18 & 4.41 & -0.80 & 0.59 & 0.0000428 & 0.0000366 & & \\
\hline \multirow[b]{2}{*}{ MT } & & & & & & & 0.0000816 & 0.0000576 & \multirow[b]{2}{*}{-34309} & \multirow[b]{2}{*}{-68567} \\
\hline & 0.00101 & 0.00067 & . & 2.35 & . & . & 0.0000576 & 0.0000499 & & \\
\hline \multirow[b]{2}{*}{ MST } & & & & & & & 0.0000841 & 0.0000593 & \multirow[b]{2}{*}{-34322} & \multirow[b]{2}{*}{-68576} \\
\hline & 0.00292 & 0.00191 & - & 2.35 & -0.35 & 0.10 & 0.0000593 & 0.0000510 & & \\
\hline & $\mu_{1}$ & $\mu_{2}$ & $\lambda$ & $\bar{\alpha}$ & $\gamma_{1}$ & $\gamma_{2}$ & \multicolumn{2}{|c|}{$\Sigma$} & Log-likelihood & BIC \\
\hline \multirow{2}{*}{ MGHYP } & 000163 & 000105 & 0,3723 & 03603 & 09044 & 00009 & 0.0002648 & 0.0001864 & \multirow{2}{*}{-34355} & \multirow{2}{*}{-68634 } \\
\hline & & & & & & & 0.0001864 & 0.0001617 & & \\
\hline
\end{tabular}

\section{RESULTS AND DISCUSSION}

Considering the values of Bayesian information criteria (BIC) in table 2 and comparing each MSGT and MST with their symmetric versions, we can see the improvement added to the fit due to inclusion of skewness parameter $\lambda$ in each model, the value of the shape parameter $\alpha=1.18$ indicating that the data is nearer to be distributed as (MSGDL) rather than as (MST) and this is reflected in the difference between BIC values corresponding to each MSGT and MST, finally we also see according to BIC that MSGT outperforms MGHYP which is very popular choice in modeling multidimensional financial data for providing great deal of flexibility.

In brief, MSGT has the potential to well fit multivariate real world data and can be compared to MGHYP which is of the same number of parameters.

\section{CONClusion AND Future Scope}

A skew extension of the multivariate generalized $\mathrm{T}$ distribution has been proposed, the new family of distributions includes many other subfamilies as special or limiting cases, this model will be useful in different contexts, we have fitted it to financial data to show its ability in capturing more features of the data by accommodating skewness after conducting a simulation study to evaluate the MLE, however, the proposed extension should be further investigated with regard to its distributional properties.

\section{REFERENCES}

[1] Azzalini A., "A class of distributions which includes the normal ones", Scandinavian journal of statistics, pp171-178, 1985.

[2] Azzalini A., Valle A. D., "The multivariate skew-normal distribution”, Biometrika, Vol.83, Issue.4, pp715-726, 1996.

[3] Azzalini A., Capitanio A., "Distributions generated by perturbation of symmetry with emphasis on a multivariate skew $t$ distribution", Journal of the Royal Statistical Society: Series B (Statistical Methodology), Vol.65, Issue.2, pp367-389, 2003.

[4] Genton M. G., Loperfido N. M., "Generalized skew-elliptical distributions and their quadratic forms", Annals of the Institute of Statistical Mathematics, Vol.57, Issue.2, pp389-401, 2005.

[5] Arslan O., "Family of multivariate generalized t distributions", Journal of Multivariate Analysis, Vol.89, Issue.2, pp329-337, 2004.

[6] McDonald J. B., Newey W. K., "Partially adaptive estimation of regression models via the generalized t distribution", Econometric theory, Vol.4, Issue.3, pp428-457, 1988.

[7] Nadarajah S., "On the generalized $t$ (gt) distribution", Statistics, Vol.42, No.5, 467-473, 2008.

[8] Box G. E., Tiao G. C., “A further look at robustness via bayes's theorem", Biometrika, Vol.49, Issue.4, pp419-432, 1962.

[9] Hoq A., Ali, M., "Templetion, estimation of parameters of a generalized life testing models", J. Statist. Res, Vol.9, pp67-79, 1974.

[10] G'omez E., Gomez-Viilegas M., Marin J., "A multivariate generalization of the power exponential family of distributions", Communications in Statistics-Theory and Methods, Vol.27, Issue.3, pp589-600, 1998.

[11] Fares A. M., Haragopal, V. V., "The generalized double Lomax distribution with applications", Statistica, Vol.76, Issue.4, pp341352, 2016.

[12] Fang K., Zhang Y., "Generalized multivariate analysis”, Science Press, Springer-Verlag, Beijing, 1990.

[13] McDonald J. B., Xu Y. J., "A generalization of the beta distribution with applications", Journal of Econometrics, Vol.66, Issue.1, pp133-152, 1995.

[14] Wang J., Boyer J., Genton M. G., "A skew-symmetric representation of multivariate distributions", Statistica Sinica, pp1259-1270, 2004.

[15] Azzalini A., "R package sn: The skew-normal and skew-t distributions (version 0.4-17)", 2011.

[16] Gupta A., "Multivariate skew t-distribution", Statistics: A Journal of Theoretical and Applied Statistics, Vol.37, Issue.4, pp359-363, 2003.

[17] Luethi D., Breymann W., "ghyp: A package on the generalized hyperbolic distribution and its special cases", R package version, Vol.1, Issue.5, 2011. 
[18] Mead M., "Generalized inverse gamma distribution and its application in reliability", Communications in Statistics-Theory and Me hods, Vol.44, Issue.7, pp1426-1435, 2015.

[19] Ruppert D., "Statistics and data analysis for financial engineering”, Springer, New York, 2011.

[20] G.V.L.N. Srihari, S.V.S.Girija, A.V. Dattatreya Rao, "On Discrete Wrapped Exponential Distribution- Characteristics", International Journal of Scientific Research in Mathematical and Statistical Sciences, Vol.5, Issue.2, pp57-64, 2018.

[21] Team R. D. C., et al., "A language and environment for statistical computing”, R foundation for statistical computing, Vienna, Austria, 2011.

[22] Theodossiou P., "Financial data and the skewed generalized $t$ distribution", Management Science, Vol.44, Issue.12, pp16501661, 1998.

\section{AUTHORS PROFILE}

Mr. M. Fares Abu Seif completed M.Sc. Applied Statistics at Osmania University, Hyderabad, India in 2015. He is currently doing a PhD in Statistics at the same University, He has published one research paper in the field of Statistics and financial data. $\mathrm{He}$ is a Faculty member of Damascus University, Syria, with over 10 years of teaching experience.

Dr V. V. HaraGopal was a former Professor of Statistics, Head and chairman Board of Statistics at Osmania University. He has guided around 20 $\mathrm{PhD}$ and $7 \mathrm{M}$. Phil students and published Around 100 papers in international and National Journals. He has delivered many invited talks across India.
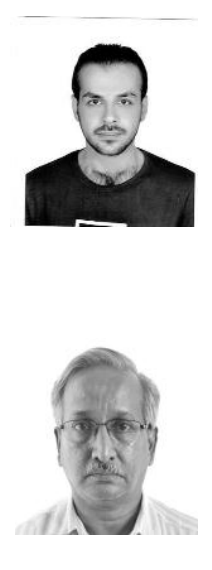